\title{
Comment on "Biotic pump of atmospheric moisture as driver of the hydrological cycle on land" by A. M. Makarieva and V. G. Gorshkov, Hydrol. Earth Syst. Sci., 11, 1013-1033, 2007
}

\author{
A. G. C. A. Meesters, A. J. Dolman, and L. A. Bruijnzeel \\ Faculty of Earth and Life Sciences, VU University, Amsterdam, The Netherlands \\ Received: 19 November 2008 - Published in Hydrol. Earth Syst. Sci. Discuss.: 16 January 2009 \\ Revised: 9 April 2009 - Accepted: 16 April 2009 - Published: 24 July 2009
}

\begin{abstract}
In their paper "Biotic pump of atmospheric moisture as driver of the hydrological cycle on land", Makarieva and Gorshkov (Hydrol. Earth Syst. Sci., 11, 1013-1033, 2007) derive from "previously unstudied" properties of atmospheric water vapor, the existence of a hitherto unknown "evaporative force". From this, a novel physical principle is deduced, according to which low-level air flows from regions with weak, to regions with strong evaporation. As such, natural forests are claimed to "suck in" moist air from the ocean, a process labeled the "biotic pump of atmospheric moisture".

This commentary focuses on the physical foundations of the Biotic Pump Theory (BPT). It is shown that the "evaporative force" on which the theory is built, is not supported by basic physical principles. The problem of moisture transport and its dependence on vegetation cover considered by Makarieva and Gorshkov is certainly important, but cannot be solved along the lines proposed by them.
\end{abstract}

\section{Introduction}

How moisture is transported from oceans to land, and the precise role of vegetation in this process, constitute key questions in our understanding of the hydrological cycle. Early modeling studies have indicated that the complete conversion of the Amazonian rain forest to pasture would lead to increased surface temperatures, a reduction in precipitation, and reduced atmospheric moisture convergence (e.g. Nobre et al., 1991; Henderson-Sellers et al., 1993). Sub-

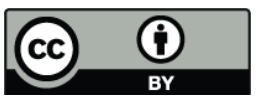

Correspondence to: A. Meesters (antoon.meesters@falw.vu.nl) sequent modeling work has by and large confirmed these results, although the magnitude of the predicted effects seems to have diminished as models became more and more sophisticated (Bruijnzeel, 2004; Costa, 2004). In addition, several modeling studies have even suggested the existence of teleconnection patterns between (Amazonian) deforestation and changes in precipitation in remote areas (e.g. Gedney and Valdes, 2000; Werth and Avissar, 2002). In most of these studies, decreased atmospheric moisture availability due to reduced soil water uptake and reduced rainfall interception after forest removal, is considered a key underlying cause of such model predictions. At the large scale, this interacts with decreased moisture convergence through reduced aerodynamic surface roughness whereas at sub-regional scales, cause and effect are complicated by meso-scale circulations (Silva Dias et al., 2002; Moore et al., 2007), subtleties in the precise triggering of convection, the generation of squall lines (idem), and effects of local topography (Dolman et al., 1999; Roy and Avissar, 2002).

Recently, a new theory has been advanced that claims a new role for forest in the generation of rainfall (Makarieva and Gorshkov, 2007; referred to in the following as M\&G). In fact, the paper by $M \& G$ has an even wider scope in that it aims to explain the causative mechanisms underlying the entire global circulation (M\&G's Sect. 3.3). Knowledge from a broad range of disciplines is combined into a coherent picture, which makes the new theory attractive to many readers and organizations interested in forest conservation or assessing the impact of land-cover change on hydrology and rainfall. However, upon closer scrutiny, it would appear that some fundamental points have been neglected by $M \& G$, whereas, in addition, the paper raises a number of issues that warrant further discussion. The basic question

Published by Copernicus Publications on behalf of the European Geosciences Union. 
addressed by $M \& G$ is: how is the land kept moistened? This question is answered by relating the theory of moisture transport and spatial distribution of precipitation to the presence or absence of (large tracts of) forest. M\&G invoke "previously unstudied" properties of atmospheric water vapor, which can be either in or out of aerostatic equilibrium ( $M \& G$, Sect. 3.1). From this, they derive a hitherto unknown "evaporative force" (M\&G, Sect. 3.2). A novel physical principle is then formulated, according to which low-level air moves from areas with weak evaporation to areas with more intensive evaporation. Next, natural forests are claimed to "suck in" moist air from the ocean, a process labeled the "biotic pump of atmospheric moisture". This "Biotic Pump Theory" (BPT) constitutes the core of their paper.

It will be shown in this commentary that the analysis of $M \& G$ is incorrect with regard to their use of certain fundamental principles of atmospheric physics. Whilst the disequilibrium on which their theory is based does exist, its effect on the transport of water vapor will be shown to be negligible, in contradiction to the results derived by M\&G. In summary, $M \& G$ 's concept of "evaporative force" will be shown to be not realistic.

\section{Two kinds of equilibrium}

\section{1 "Traditional" theory of equilibrium}

There are two kinds of equilibrium to be distinguished, and it is critically important not to confuse the two. The first, which we shall call "bulk-equilibrium" for convenience sake, is the well-known hydrostatic equilibrium, i.e. the pressure difference over a vertical column of air equals the weight per area of the column (e.g. Landau and Lifshitz, 1987) and no vertical motion occurs:

$-\frac{\partial p}{\partial z}=\rho g$

where $z=$ height, $\quad p=$ pressure, $\rho=$ mass density and $g=$ acceleration due to gravity. This equation is fundamental in meteorology in that deviations from equilibrium cause a vertical motion according to the vertical Euler equation:

$\rho \frac{d w}{d t}=-\frac{\partial p}{\partial z}-\rho g$

(where $w=$ vertical velocity, positive when upward; and $t=$ time) which will continue until equilibrium is restored.

Equations (1) and (2) are mechanical equations which apply to air as such (not its separate components), and they determine the motion of air parcels as a whole. For a homogeneous mixture, hydrostatic equilibrium corresponds to a density profile which can be derived by combining Eq. (1) with the equation-of-state:

$p=\rho(R / M) T$ (where $R=$ universal gas constant per mol, $M=$ mean mass per mol of the mixture, and $T=$ absolute temperature). Assuming $T$ to be constant for convenience, this leads to a height distribution:

$\rho(z)=\rho_{\text {sur }} \exp (-z / h)$

in which $\rho_{\text {sur }}$ is the density for $z=0$, and $h$ is the scale height for the mixture:

$h=\frac{R T}{M g}$

Upon substituting $M=29 \mathrm{~g} \mathrm{~mol}^{-1}$ (the value for dry air) and $T=288 \mathrm{~K}$ one obtains $h=8.4 \mathrm{~km}$.

Air consists of components which may be indicated with the subscript $i$. The mass density $\rho$ is then the sum of the densities of the components $i$. Further, Dalton's law states that the pressure $p$ is the sum of the partial pressures $p_{i}$ of the components, for which the equation-of-state is:

$p_{i}=\rho_{i}\left(R / M_{i}\right) T$

The second kind of equilibrium is thermodynamic equilibrium. We will call this "component-equilibrium" for convenience, since it applies to each component of the mixture separately. According to Boltzmann's equation, if $T$ is constant the thermodynamic equilibrium density of the $i$-th component is given by:

$\rho_{i}(z)=\rho_{i, \text { sur }} \exp \left(-\frac{E_{i}(z)}{R T}\right)$

in which $E_{i}(z)=M_{i} g z$ or the potential energy per mol due to gravitation (Landau and Lifshitz, 1987, paragraph 59). This distribution can also be written as:

$\rho_{i}(z)=\rho_{i, \text { sur }} \exp \left(-z / h_{i}\right)$

in which the scaling height $h_{i}$ is given by:

$h_{i}=\frac{R T}{M_{i} g}$

with $M_{i}$ the molar mass of the $i$-th constituent. Equations (8) and (9) are similar to Eqs. (4) and (5), but now every component has its own scaling height. For water vapor at $T=288 \mathrm{~K}$, $h_{i}=13.5 \mathrm{~km}$, which is much larger than that for nitrogen or oxygen: viz. $8.7 \mathrm{~km}$ and $7.6 \mathrm{~km}$, respectively. Using the equation-of-state per component Eq. (6), the component equilibrium profile can be shown to satisfy the counterpart of Eq. (1):

$-\frac{\partial p_{i}}{\partial z}=\rho_{i} g$

However, this component-equilibrium should not be called "hydrostatic" or "aerostatic" equilibrium, and is not to be thought of in mechanical terms (such as partial pressures being in balance with the weights of the respective components), as is sometimes done by M\&G (e.g. in the beginning of their Sect. 3.1). It must be emphasized that the 
component-Eqs. (8, 9, and 10) are valid only for thermodynamic equilibrium.

Whilst bulk-equilibrium is restored by macroscopic motion, restoring of (thermodynamic) component-equilibrium requires a process in which the components move separately. The only process capable of this is (molecular) diffusion (Landau and Lifshitz, 1987, Sect. 57). However, this is a very slow process, compared with macroscopic atmospheric transport mechanisms as molecular diffusion coefficients in the lower troposphere are in the order of ca. $10^{-5} \mathrm{~m}^{2} \mathrm{~s}^{-1}$ (Tennekes and Lumley, 1990). The relation between molecular diffusion and large-scale thermodynamic equilibrium under an external force, first elucidated by Einstein, has been described by Van Kampen (1983), Dill and Bromberg (2003), and (very briefly) by Landau and Lifshitz (1987), Sect. 59. The result is that the equilibrium-restoring diffusive flux $F_{i}$ (positive when upward) can be expressed in the terminology of $M \& G$ as:

$F_{i}=-v_{i}\left(\frac{\partial \rho_{i}}{\partial z}+\frac{\rho_{i}}{h_{i}}\right)$

where $v_{i}$ is the molecular diffusivity. The first term represents the common Fick's Law, whereas the second term is a modification due to the acting of gravity on the molecules.

What then are the practical consequences of these two equilibria? These are very different for (hydrostatic) bulk-equilibrium on the one hand, and (thermodynamic) component-equilibrium on the other. Deviations from bulkequilibrium cause macroscopic motions which act to restore equilibrium in a highly efficient manner. It is well known (Wallace and Hobbs, 1977; Holton, 1979; Dutton, 1986; and many others) that air is usually in hydrostatic equilibrium, to a very good approximation, except when local phenomena such as up- and down-drafts occur.

There are several mechanisms that bring about this rapid restoration. First of all, local distortion of equilibrium generates an elastic relaxation of the surrounding air that is propagated with the speed of sound (Lamb, 1945, chapter 10; Bannon, 1995; Van Delden, 2000). The ensuing displacements are generally very small and hard to detect, although for special cases (e.g. the continental sea breeze effect) the involved horizontal displacements cause a detectable change in surface pressure which also moves at the speed of sound (Tijm and Van Delden, 1999). To the extent that this fast relaxation is insufficient to restore equilibrium, gravity waves or convection currents will arise, depending on whether the air is stably or unstably stratified. Unlike elastic relaxation, these mechanisms cause systematic flow over large distances, hence they have received far more attention in the literature.

On the other hand, deviations of component-equilibrium cannot cause restoring motions, only diffusive fluxes which are very weak (Eq. 11). Because component-disequilibrium has so little effect, it is barely considered in atmospheric science as a causative factor, except in relation to interface- processes which always act on the micro-scale (e.g. evaporation at a surface, cloud microphysics).

\subsection{The approach of M\&G to the two kinds of equilibrium}

The key question in the context of the "evaporative force" postulated by $M \& G$ is whether component-equilibrium is of importance to macroscopic transport in the atmosphere. $M \& G$ deny this correctly for water vapor: observed vapor profiles are much more compressed than the equilibriumprofile predicted by Eq. (8) using $h_{i}=13.5 \mathrm{~km}$ at $T=288 \mathrm{~K}$. The reason is that vapor is continuously entering the atmosphere at the surface by evaporation, whereas it is removed at greater altitudes by condensation and precipitation. This is traditional knowledge, and the BPT is not required to explain this deviation from non-condensing gases.

However, for dry air, there is a complication. Dry air consists of several components, each with a different molar mass and consequently with different scaling heights. M\&G accept the fact that these components are well-mixed in the troposphere (McEwan and Phillips, 1975; Wallace and Hobbs, 1977), and thus that dry air has a near-constant composition. Hence, it can be treated as a single component, with a molar mass of $M_{d}=29 \mathrm{~g} \mathrm{~mol}^{-1}$ (obtained by averaging the respective components; $M \& G$, Sect. 3.1).

Next, M\&G make a very important assumption, stating that for the dry air $(d)$ component, component-equilibrium should always hold:

$-\frac{\partial p_{d}}{\partial z}-\rho_{d} g=0$

It should be noted first of all that this assumption is in sharp contradiction to the "traditional" assumption that it is the bulk-equilibrium rather than the component-equilibrium that is maintained. The reason is that bulk-equilibrium is linked to dry air $(d)$ equilibrium and vapor $(v)$ equilibrium by:

$$
-\frac{\partial p}{\partial z}-\rho g=\left(-\frac{\partial p_{d}}{\partial z}-\rho_{d} g\right)+\left(-\frac{\partial p_{v}}{\partial z}-\rho_{v} g\right)
$$

Since it is taken for granted that the vapor component is not in equilibrium, acceptance of Eq. (12) implies that there is no bulk-equilibrium either. This consequence is already announced in M\&G's Sect. 3.1 ("Moist air does not conform to either Eq. 7 or Eq. 8"), and is applied in M\&G's Sect. 3.2 after M\&G's Eq. (10). It constitutes a key building block in the derivation of M\&G's concept of "evaporative force".

Equation (1) follows immediately from the impossibility to distinguish physically between the effect on the pressure within an air parcel of, respectively, the water vapor molecules and the "dry air" molecules. It is unclear why $M \& G$ have replaced this "traditional" assumption of bulk-equilibrium (Eq. 1) with an assumption of componentequilibrium for dry air (Eq. 12). The attribution of M\&G's Eq. (7) to Landau and Lifshitz (1987) appears inappropriate. 

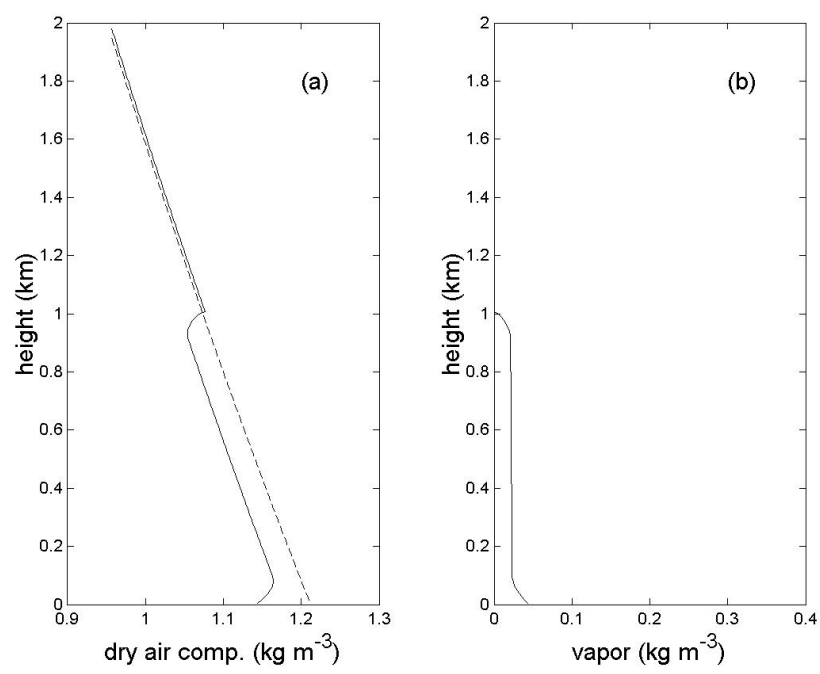

Fig. 1. Illustration of the reaction of a dry boundary layer to evaporation. (a) Dashed line: dry air mass density, before evaporation. Solid line: the same, after evaporation. (b) Added water vapor mass density (exaggerated).

One consideration is that, unlike for water vapor, the equilibrium is not affected by inflow and outflow of the component. Also, we have already noted that $M \& G$ speak in a confused way about component-equilibrium as "hydrostatic equilibrium". Furthermore, the following citation from $M \& G$ seems key in this respect:

"In agreement with Dalton's law, partial pressures of different gases in a mixture independently come in or out of the equilibrium. The non-equilibrium state of atmospheric water vapor cannot bring about a compensating deviation from the equilibrium of other gases..." (start of new paragraph on p. 1022).

Interestingly, this line of thinking is remarkably similar to that found in the once widely used textbook (in German) on meteorology by Von Hann (1915):

"According to Dalton's Law, the distribution of a gas is independent of the distribution of another gas in the same space (unless chemical reactions occur). Another gas may retard its spreading out, but not its final distribution. Kinetic gas theory has proven this law (by Boltzmann). (...). Thus, the atmosphere can be considered as composed of several independent atmospheres, i.e. an autonomous nitrogen, oxygen and argon atmosphere." (Sect. I-c).

This principle might be appropriate for certain situations in which macroscopic flows can be excluded (e.g. in the laboratory), but it appears inappropriate for open-air conditions where macroscopic flows are so dominant that componentequilibrium becomes of marginal importance. For example, contrary to the prediction of Eq. (8), the observed dry-air composition is constant in the troposphere as a consequence of the strong vertical mixing induced by upward and downward motions. We have seen earlier that $M \& G$ accept the homogenization of the dry air components, but surprisingly they do not consider the effect of macroscopic motions when formulating their interpretation of Dalton's Law. If macroscopic motion is included (as should practically always be done when considering atmospheric matters) separate behavior of the components becomes untenable. In particular, the efficient way in which bulk-equilibrium tends to be restored by macroscopic flow, implies that any disequilibrium for one component would tend to be compensated by disequilibrium for other components according to Eq. (13). Below we will illustrate this for the special case of evaporation, as $M \& G$ their "evaporative force" depends critically on this part of the theory.

\section{Application to evaporation}

\subsection{Immediate influence of evaporation on atmospheric dynamics: "traditional" theory}

Let us assume a flat surface and consider quantities which are averages over a large horizontal area (e.g. vertical velocity $w$ is an average that will be smaller than the positive and negative $w$-values reached in turbulent air). Let us assume further that initially there is only dry air, with its vertical density distribution $\rho_{d}(z)$ in equilibrium (Fig. 1a). Dry air is treated as a single component for convenience. While the surface is evaporating, the local pressure rises slightly due to the additional vapor molecules, and consequently the column above (which still has the same weight) is being lifted slightly. Consequently, the lower layer is expanding - to make room for the water molecules - so that its pressure is brought again in equilibrium with the weight of the column above. A simple calculation shows that for an evaporation rate $E=1 \mathrm{~mm} \mathrm{~h}^{-1}$ (which is considerable) the lifting velocity will be about $0.4 \mathrm{~mm} \mathrm{~s}^{-1}$ if $p=10^{5} \mathrm{~Pa}$ and $T=288 \mathrm{~K}$. Since the incurred acceleration $d w / d t$ will be very small, it follows from Eq. (2) that during the entire process, hydrostatic equilibrium will be approximately maintained. Note in passing that, on the other hand, condensation would cause compression of the air (neglecting thermal effects).

What are the consequences of the above for the equilibrium of separate components? Certainly, the water vapor (Fig. 1b) will not reach equilibrium since this would require the water vapor to be taken up to very great height (Eq. 8 with $\left.h_{v}=13.5 \mathrm{~km}\right)$. We agree with M\&G that observed $\rho_{v}$-profiles are usually compressed vertically with respect to the equilibrium profile. But will the equilibrium for the dry-air profile be preserved, as M\&G claim? Above the boundary layer, the profile is raised to a higher level (Fig. 1a), but density values will still correspond with their original pressure levels, thereby preserving the original profile in a sense. However, within the boundary layer, expansion has occurred to make room for the water molecules added by surface evaporation and this must have caused the dry-air component 
to depart from its original equilibrium (according to Eq. 8, Fig. 1a). Thus, a deviation from equilibrium for one component is transferred automatically to another through macroscopic motion, in this case the expansion of the boundary layer. Disequilibrium for one air component cannot coexist for long with equilibrium of the other components, since that would mean (according to Eq. 13) bulk disequilibrium, and hence the initiation of restoring motion.

\subsection{M\&G's approach to evaporation}

We have now reached a point at which we can evaluate $M \& G$ 's derivation of the "evaporative force". It follows from Eqs. (2) and (13) that:

$\rho \frac{d w}{d z}=\left(-\frac{\partial p_{d}}{\partial z}-\rho_{d} g\right)+\left(-\frac{\partial p_{v}}{\partial z}-\rho_{v} g\right)$

$M \& G$ assume stationary motion, so that because of:

$$
\frac{d w}{d t}=\frac{\partial w}{\partial t}+w \frac{\partial w}{\partial z}
$$

the left-hand side of Eq. (14) may be replaced by $\frac{1}{2} \rho \partial w^{2} / \partial z$. In the "traditional" view (Sect. 3.1), the vertical velocity $w$ is very low, and therefore the acceleration in the left-hand side of Eq. (14) is very weak. Consequently, the two terms on the right-hand side should almost cancel each other (hence there is bulk-equilibrium). However, at this point $M \& G$ assume equilibrium for the dry-air component (Eq. 12) and thus their version of Eq. (14) becomes:

$\rho \frac{d w}{d t}=-\frac{\partial p_{v}}{\partial z}-\rho_{v} g$

This is M\&G's Eq. (15). As the vapor-disequilibrium on the right-hand side is now - incorrectly - no longer balanced by the dry-air disequilibrium, there is consequently a very strong deviation from bulk-equilibrium which is bound to lead to a very violent restoring motion. A numerical example (M\&G's Eq. 18) yields vertical velocities of as much as $50 \mathrm{~m} \mathrm{~s}^{-1}$, considered by them to be "in good agreement with the maximum updraft velocities observed in typhoons and tornadoes". However, according to the M\&G theory such stationary velocities should be common above any evaporating surface!

The critical issue neglected by $M \& G$ is the response of the dry-air component to the motions caused by the bulkdisequilibrium. Even if Eq. (16) would be true initially, such violent motions would cause a very rapid expansion of the air column (thereby restoring bulk-equilibrium) while at the same time disturbing the equilibrium for the dry-air component. However, M\&G imagine an atmosphere in which the dry-air component stays immobile in the presence of (even violent) vertical motion. This logical contradiction leads to an atmosphere in which bulk-equilibrium cannot be restored.

It may be elucidating at this point to consider a further thought experiment. Consider a container filled with a boundary layer consisting of dry air, which is then covered with an air-tight lid. Next, water vapor with a realistic vertical profile is added to the container, without lifting the lid. The air cannot expand, due to the closed lid, hence $\rho_{d}$ will remain approximately the same. This will cause a substantial overpressure. Upon pulling the lid, elastic relaxation will occur in the form of a sudden expansion (in which initially a very high acceleration as described by Eq. 16 is involved), but this will be a transient phenomenon as mechanical equilibrium is soon restored (as per common experience!). After this, the moist air has the same $p$, but still a higher $p_{v}$ than the background air. Consequently, since $p_{d}$ equals $p-p_{v}$, $p_{d}$ must have become lower than that of the background air. This implies a violation of $M \& G$ their so-called fundamental principle that "components independently come into and out of equilibrium".

The strong upward force implied by Eq. (16) is called "evaporative force" by M\&G. Because surface evaporation is far too small to provide the very strong upward flow of vapor in the atmosphere presumed by them, $M \& G$ argue that lateral inflow of vapor by advection is needed to restore the vapor mass balance. However, a more careful ("traditional") analysis of the motion associated with evaporation as presented here shows no reason for such a discontinuity. The motion caused by evaporation leads to an expansion of the boundary layer which is just enough to make room for the water vapor added by the evaporation, and this involves no disturbance of the mass balance.

The inferred horizontal inflow caused by the "evaporative force" is worked out further in M\&G's Sect. 3.3. The very strong force predicted by the original equations to be acting upon the vertical column, is now thought to be redistributed over a flow trajectory that includes a long horizontal stretch, so that the pressure gradients and the resulting accelerations become much weaker. However, this causes a conflict with the original equations, and especially with the principle that disequilibrium of the vapor component cannot cause a compensating disequilibrium of the dry air component. Finally, $M \& G$ express the power of the evaporative force using a new characteristic velocity $w_{f}$. It is pertinent to note that the typical value for $w_{f}$ of $5.6 \mathrm{~mm} \mathrm{~s}^{-1}$ derived by $\mathrm{M} \& \mathrm{G}$, is four orders of magnitude smaller than the value given by $M \& G$ in their Eq. (18). Apparently this is necessary to bring their theory into agreement with the observations.

There is another point that should draw our attention. It would follow from consequent application of the "evaporative force" theory that the oceans, being mostly stronger evaporators than the continents, should themselves "suck" moisture from the air, and this would cause horizontal flows of moisture at the expense of the moisture supply of the land, directly opposing the BPT. It is remarkable that this point is not at all considered by $M \& G$. 


\section{What drives the atmospheric circulation?}

The following short section has been added to remove any potential confusion about the mechanism behind atmospheric circulation, which is treated at length in Sect. 3.3 of M\&G. Whilst very brief, this can easily be supplemented by consulting textbooks such as Anthes (1997) and many others.

The atmospheric circulation is driven by pressure gradients together with the Coriolis force (due to the rotation of the Earth). Pressure differences are caused by the different weights of the air column in different places, which are caused in turn by temperature differences mostly. The latter have two principal origins: (1) surface differential heating, and (2) condensation (a process in which heat is released). The second process occurs mainly in air that is being lifted already by thermally-driven circulations. The heat that is released upon vapor condensation causes local expansion of the air, such that it becomes lighter than dry air at the same height. Condensation also involves removal of water from the vapor phase and although this process acts in the opposite direction, the effect is only minor. Overall, condensation tends to strengthen the already existing convective circulations by making the air locally lighter.

On a somewhat longer term, the circulation of water can have a dampening effect on the atmospheric circulation: clouds diminish surface heating (during daytime) and cooling (at night), and evaporation from the surface consumes energy at the expense of the sensible heat flux. Hence condensation and evaporation both influence atmospheric circulation in several ways, but their influence is secondary compared to that exerted by differential heating at the surface. Moreover, their influence does not work along the lines proposed by M\&G.

The fact that regions with natural forest vegetation are also regions of high precipitation, is not necessarily proof of a complete redirection of atmospheric circulation by the forest (although there may be some influence). Rather, it is the atmospheric circulation that determines where forests can grow. As explained in the introduction, there is some evidence that the presence of extended tracts of forest (e.g. in the humid tropics) promotes inland transport of moisture. The most important mechanism in this regard seems to be the recycling of moisture by the forest, for which isotopic evidence was found in the Amazon basin (Salati and Vose, 1984).

As an example of the application of their theory, $M \& G$ have suggested that the high precipitation received by the Yenisey basin in central Siberia is the result of substantial moisture transport from the Arctic Sea (where the air is cold and very dry) deep into the continent and invoked by the existence of their "Biotic Pump". However, inspection of the meteorological data shows that the moisture is advected by air currents coming from the west rather than from the north, mostly from relatively warm sources such as the Mediterranean Sea and the Atlantic Ocean (Lydolph, 1977). This general flow from the west is adequately explained by tradi- tional theory in terms of the prevailing gradient in zonal temperatures plus the Coriolis force, and therefore does not require the existence of a "Biotic Pump". The enhanced precipitation in the Yenisey basin is further caused by orographic lifting of the air as it enters the Central Siberian Plateau. Nevertheless, the long distance over which this moisture is transported implies considerable recycling of precipitation (Lydolph, 1977), and it is likely that the forest plays a crucial role in this regard. However, this role lies in re-evaporation of moisture, and not in the complete redirection of the general circulation.

In summary, high precipitation occurring at sites located far from the ocean can be explained adequately by traditional theory, whereas in the case of central Siberia the predictions by the theory of M\&G (advection from the north) are in conflict with observations (advection from the west).

\section{Concluding remarks}

In this commentary the theoretical basis of the "evaporative force" proposed by Makarieva and Gorshkov (2007, M\&G) to explain large-scale precipitation gradients in relation to the presence or absence of forest vegetation has been analyzed in some detail. It is concluded that M\&G's theory is based on an incorrect interpretation of basic physical principles operating in a free atmosphere. However, it should be emphasized that this commentary is more limited in scope than the paper by M\&G. For example, it does not address the problems of moisture transport and the spatial distribution of precipitation, as summed up in the valuable introductory part of $M \& G$ that draws attention to the various phenomena requiring further study. At the same time, $M \& G$ do not do full justice to the existing literature; in particular, they ignore such complex spatio-temporal atmospheric flow patterns as the ascending and descending branches of the Hadley Circulation, the shielding effect of mountain ranges, organized convection around forested islands, etc., all of which are fundamental to understanding precipitation regimes and vegetation zonation (cf. Walter, 1964; Walter and Lieth, 1967; Van der Molen et al., 2006).

The question as to whether or not the existence of some kind of "biotic pump" is necessary to explain certain observed phenomena is also outside the scope of this commentary, but we do believe with $M \& G$ that the role of vegetation - and in particular forest - in generating rainfall is still poorly understood. Likewise, with changes in terrestrial land cover due to deforestation being on the increase, such questions potentially assume added importance. $M \& G$ are to be complimented for their valiant attempt to shed more light on the interaction between forest vegetation and precipitation. However, a good understanding of these phenomena should be based on well-founded scientific principles, and not be supported by arguments that are demonstrably physically incorrect. 
Acknowledgements. This investigation was funded by the EU FP6 project Water and Global Change (WATCH) Program.

Edited by: B. van den Hurk

\section{References}

Anthes, R. A.: Meteorology, 7nd ed., Prentice Hall, New Jersey, 1997.

Bannon, P. R.: Hydrostatic adjustment: Lamb's problem, J. Atm. Sci., 52, 1743-1752, 1995.

Bruijnzeel, L. A.: Hydrological functions of tropical forests: not seeing the soil for the trees?, Agr. Ecosyst. Environ., 104, 185228, doi:10.1016/j.agee.2004.01.015, 2004.

Costa, M. H.: Large-Scale Hydrological Impacts of Tropical Forest Conversion, in: Forests, Water and People in the Humid Tropics, edited by: Bonell, M. and Bruijnzeel, L. A., Cambridge University Press, Cambridge, 590-597, 2004.

Dill, K. A. and Bromberg, S.: Molecular driving forces, Garland Science, New York, 2003.

Dolman, A. J., Silva Dias, M. A. F., Calvet, J-C., Ashby, M., Tahara, A. S., Delire, C., Kabat, P., Fisch, G. F., and Nobre, C. A.: Meso-scale effects of tropical deforestation in Amazonia: preparatory LBA modelling studies, Ann. Geophys.-Italy, 17, 1095-1110, 1999.

Dutton, J. A.: The Ceaseless Wind, an Introduction to the Theory of Atmospheric Motion, Dover Publications, New York, 1986.

Gedney, N. and Valdes, P. J.: The effect of Amazonian deforestation on the Northern Hemisphere circulation and climate, Geophys. Res. Lett., 27(19), 3053-3056, 2000.

Henderson-Sellers, A., Dickinson, R. E., Durbidge, T. B., Kennedy, P. J., McGuffie, K., and Pitman, A. J.: Tropical deforestation - Modeling local-scale to regional-scale climate change, J. Geophys. Res., 98(D4), 7289-7315, 1993.

Holton, J. R.: An Introduction to Dynamic Meteorology, 2nd edn., Academic Press, New York, 1979.

Lamb, H.: Hydrodynamics, 6th edition, Dover, New York, 1945.

Landau, L. D. and Lifshitz, E. M.: Course of Theoretical Physics, 6, Fluid Mechanics, Pergamon Press, Oxford, 1987

Lydolph, P. E.: Climates of the Soviet Union. World Survey of Climatology, volume 7, Elsevier, Amsterdam-Oxford-New York, 1977.

Makarieva, A. M. and Gorshkov, V. G.: Biotic pump of atmospheric moisture as driver of the hydrological cycle on land, Hydrol. Earth Syst. Sci., 11, 1013-1033, 2007, http://www.hydrol-earth-syst-sci.net/11/1013/2007/.
McEwan, M. J. and Phillips, L. F.: Chemistry of the Atmosphere, Edward Arnold, London, 1975.

Moore, N., Arima, E., Walker, R., and Ramos da Silva, R.: Uncertainty and the changing hydroclimatology of the Amazon, Geophys. Res. Lett., 34, L14707, doi:10.1029/2007GL030157, 2007.

Nobre, C. A., Sellers, P. J., and Shukla, J.: Amazonian deforestation and regional climate change, J. Climate, 4, 957-988, 1991.

Roy, S. B. and Avissar, R.: Impact of land use/land cover change on regional hydrometeorology in Amazonia, J. Geophys. Res., 107(D20), 8037, doi:10.1029/2001JD000662, 2002.

Salati, E. and Vose, P. B.: Amazon Basin - A system in equilibrium, Science 225, 129-138, 1984.

Silva Dias, M. A. F., Rutledge, S., Kabat, P., Silva Dias, P. L., Nobre, C., Fisch, G., Dolman, A. J., Zipser, E., Garstang, M., Manzi, A. O., Fuentes, J. D., Rocha, H. R., Marengo, J., Plana-Fattori, A., Sá, L. D. A., Alvalá, R. C. S., Andreae, M. O., Artaxo, P., Gielow, R., and Gatti, L.: Cloud and rain processes in a biosphere atmosphere interaction context in the Amazon Region, J. Geophys. Res., 107(D20), 8072, doi:10.1029/2001JD000335, 2002.

Tennekes, H. and Lumley J. L.: A First Course in Turbulence, The MIT Press, Cambridge MA, 1990.

Tijm, A. B. C. and Van Delden, A. J.: The role of sound waves in sea breeze initiation, Q. J. Roy. Meteorol. Soc., 125, 1997-2018, 1999.

Van Delden, A.: Linear dynamics of hydrostatic adjustment to horizontally homogeneous heating, Tellus, 52B, 380-390, 2000.

Van Kampen, N. G.: Stochastic Processes in Physics and Chemistry, North Holland Publishing Company, Amsterdam, 1983.

Van der Molen, M. K., Dolman, A. J., Waterloo, M. J., and Bruijnzeel, L. A.: Climate is affected more by maritime than by continental landuse change: a multiple-scale analysis, Global Planet. Change, 54, 128-149, 2006.

Von Hann, J.: Lehrbuch der Meteorologie, Verlag von Christian Hermann Tauchnitz, Leipzig, 1915.

Wallace, J. M. and Hobbs, P. V.: Atmospheric Sciences, an Introductory Survey, Academic Press, Orlando FL, 1977.

Walter, H.: Die Vegetation der Erde in oeko-physiologischer Betrachtung, Fisher, Jena, 1964.

Walter, H. and Lieth, H.: Klimadiagramm Weltatlas, Gustav Fisher, Jena, 1967.

Werth, D. and Avissar, R.: The local and global effect of Amazon deforestation, J. Geophys. Res., 107(D20), 8087, doi:10.1029/2001JD000717, 2002. 